\title{
Bericht über Teuto-Brasilianisch, Plautdietsch und Namsläng: Spuren und Einflüsse von Migration aus dem deutschsprachigen Raum. Lehrstuhl für Germanistik der Babes-Bolyai Universität
}

\author{
Cluj-Napoce, 2018
}

\section{Erkan Osmanović}

Zwischen dem 25. und 26. Jänner 2018 fand in der rumänischen Stadt Cluj-Napoce (Klausenburg) die internationale Tagung „Teuto-Brasilianisch, Plautdietsch und Namsläng: Spuren und Einflüsse von Migration aus dem deutschsprachigen Raum " in der Österreich-Bibliothek der Babes-Bolyai Universität statt. Die Konferenz entstand aus einer Zusammenarbeit folgender Institutionen: Babes-Bolyai Universität, dem Österreichischen Kulturforum Bukarest, dem DAAD (Deutscher Akademischer Austauschdienst) und den Österreich-Bibliotheken. Das Organisationsteam setzte sich aus Veronika Zwing (OeAD-Lektorin), Franziska Schwantuschke (DAAD-Lektorin) und der DAAD-Sprachassistentin Michalea Blume.

Die Tagung ging diversen Folgen nach: (1) welche Fälle von prominenter Immigrationsbewegung deutschsprachiger Menschen gibt es aus historischer und aktueller Persepktive; (2) auf welche Art und Weise deutschsprachige Immigration eine Gesellschaft, ihre Kultur und Sprache verändern konnte oder kann; (3) welche Spuren und Veränderungen diese in den Aufnahmegesellschaften in Kunst, Architektur, Literatur und Sprachen hinterlassen haben? (4) Welche Rück- und Wechselwirkungen gab es wiederum auf und mit den Ursprungsländern; (5) welche Schlüsse können daraus gezogen werden?

Die Tagung selbst wurde durch eine Rede des Prorektor und wissenschaftlichen Leiters der Österreich Bibliothek Cluj-Napoce Univ.-Prof. Dr. Rudolf Gräf eröffnet. Danach übernahm Veronika Zwing, MA das Wort und skizzierte in ihrem Beitrag Deutschland, Österreich und die Schweiz als Emigrationsländer einerseits die Verbindungslinien der einzelnen Beiträge, andererseits brachte sie die Thematik der Tagung auf den Boden tagesaktueller politischer Veränderungen innerhalb Europas - man denke etwa an den problematischen Umgang der Europäischen Union mit der gesteigerten Anzahl an Geflüchteten aus dem Nahen Osten und Nordafrika. 
Danach begann der erste Tag der Tagung, dessen Schwerpunkt in der Immigration deutschsprachiger Menschen innerhalb Europas lag.

Sabina de Carlo beschäftigte sich in ihrem Beitrag Wohin steuert die deutsche Sprache im Banat? Spracheinstellungen als Gradmesser für eine Entwicklung mit einer Untersuchung des Deutschen unter Germanistik-Studierenden in Rumänien. Dabei wurden die Studierenden zu ihrer Einstellung gegenüber der deutschen Sprache befragt, etwa inwiefern sie der Sprache affirmativ oder ablehnend gegenüberstehen und worauf sie dies zurückführen würden (u.a. Laut, biographische Erlebnisse). Danach thematisierte Edit Szegedi in ihrem historisch ausgerichteten Vortrag Deutsch als Sprache der Bürger, Nonkonformisten und Flüchtlinge im frühneuzeitlichen Klausenburg die Auswirkungen der deutschsprachigen Einflüsse in den theologischen Schriften Clujs und deren gleichzeitigem Transfer der Ansichten der Täuferbewegung.

Nun folgte ein Sprung in die literaturwissenschaftliche Betrachtung deutschsprachiger Immigration. Kinga Borsos nahm sich in ihrem Beitrag Die Rolle von psychologischen Motiven bei Herta Müller und Terézia Mora nicht nur den Biographien der beiden Autorinnen an, sondern fand darüber hinausgehend auch Aus- und Wechselwirkungen der eigenen Biographie in den jeweiligen literarischen Texten.

Im Anschluss hieran folgte mein Vortrag, der sowohl literaturgeschichtlich, als auch histographisch ausgerichtet war und sich mit dem Leben der beinahe vergessenen Autorin und Journalistin Milena Mrazović beschäftigte. Das österreichisch-bosnische Bild der Milena Mrazović nahm sich der lückenhaften Biographie der Autorin an und ihrer Rolle als Leiterin der in Bosnien-Herzegowina erschienen deutschsprachigen Zeitschrift Die bosnische Post und der möglichen Wirkungsstärke ihrer Artikel.

Den Abschlussbeitrag des ersten Tages lieferte Heinrich Siemens, der sich in seinem Beitrag Plautdietsch und seine Umgebungssprachen Deutsch, Russisch, Englisch, Spanisch und Portugiesisch der historischen Ausbreitung des Plautdeutschen in Europa und Südamerika, und deren aktueller Situation in südamerikanischen Staaten widmete.

Der erste gemeinsame Abend wurde durch eine offene Gesprächsrunde abgerundet. Unter dem Titel Aus Geschichte und Alltag deutschsprachiger RumänInnen fanden sich Siebenbürger Sachsinnen und Sachsen verschiedener Generationen zusammen, die ihre eigene (Familien-)Geschichte und ihren Alltag als Deutschsprachige in Rumänien berichteten.

Der zweite Tagungstag startete mit einem ausgedehnte Stadtspaziergang durch den historischen Kern der Altstadt Cluj-Napoces; dabei griff Edit Szegedi auf diverse Punkte der Vorträge des vorigen Tages auf und machte sie anhand von diversen Gebäude und Plätzen nachvollziehbarer.

Danach fing der zweite Block der Konferenz erneut in den Räumen der Österreich-Bibliothek an.

Angela Abmeier und Christian E. Rieck fokussierten sich in ihrem Beitrag Best of Both Worlds? The German Community in Argentina between Assimilation and Assertiveness (1854-1955) auf Einflüsse der deutschsprachigen Gemeinden in den diversen Landesbereichen Argentiniens. Der Blick begann bei Auswirkungen innerhalb der argentinischen Architektur, ging über die Oszillation der Argentinierinnen und Argentinier zwischen den Polen Assimilation und Integration, hin zu der Aufteilung der Gemeinden in eigene 
Subgruppen, wie etwa deutschsprachige Geflüchtete der politischen Extreme im Zuge der Geschehnisse Deutschlands während des 20. Jahrhunderts.

Danach kam es mit Oliver Niels Völkels Ausführungen unter dem Titel Sprache und Integration in der deutschbrasilianischen Literatur wiederum zu einem literaturhistorischen Ausblick auf die Einflüsse der Immigrationsbewegungen.

Michael John blieb mit seinem darauffolgenden Vortrag auf demselben Kontinent, schob allerdings den Fokus weiter Richtung Norden. Er unternahm mit seinem Beitrag Donau-Blues am Hudson River. Bekannte und unbekannte Facetten des österreichischen Exils in New York einen historischen Blick auf die Ursprünge, Entwicklungen und aktuelle Lage der schwindenden Gemeinde.

Danach zeichnete Giorgia Sogos unter dem Titel Im Exil - die ferne Heimat: Vergangenheit und Gegenwart bei Adrienne Thomas einerseits das Leben der Autorin, die auf der Flucht vor den Nazis über die Schweiz, Frankreich und Österreich in die USA, und nach Kriegsende wieder zurück nach Österreich kam, nach und verwies andererseits auf die Auswirkungen dieser Fluchterfahrung in ihrem Werk.

Im letzten Vortrag nahm sich René Demanou mit Migration, Gedächtnis und Identitätsvorstellung der deutschsprachigen Minderheit in Namibia dargestellt am Beispiel von Jürgen Leskiens Dunkler Schatten Waterberg: Afrikanische Nachtgespräche (2004) eines aktuellen Werkes an und untersuchte den Gesprächsband mittels wissenschaftlicher Methodik und Systematisierung.

Als Abschluss der Tagung diente eine zusammenfassende Diskussion, die aufzeigen konnte, welches Potential sich in einer weiteren interdisziplinären Herangehensweise des Phänomens der deutschsprachigen Immigration freilegen lassen würde.

Erkan Osmanović / 238087@mail.muni.cz

Masarykova univerzita, Filozofická fakulta, Ústav germanistiky, nordistiky a nederlandistiky Arna Nováka 1, 60200 Brno, CZ 
